\title{
Le modèle Bassidji (Partie 2)
}

\section{Farhad Khosrokhavar}

\section{(2) OpenEdition}

1 Journals

\section{Édition électronique}

URL : http://journals.openedition.org/conflits/682

DOI : $10.4000 /$ conflits.682

ISSN : 1777-5345

Éditeur :

CCLS - Centre d'études sur les conflits lilberté et sécurité, L'Harmattan

\section{Édition imprimée}

Date de publication : 15 octobre 1998

ISSN : 1157-996X

\section{Référence électronique}

Farhad Khosrokhavar, «Le modèle Bassidji (Partie 2) », Cultures \& Conflits [En ligne], 29-30 | automnehiver 1998, mis en ligne le 16 mars 2006, consulté le 30 mars 2021. URL : http://

journals.openedition.org/conflits/682; DOI : https://doi.org/10.4000/conflits.682

Ce document a été généré automatiquement le 30 mars 2021.

Creative Commons License 


\title{
Le modèle Bassidji (Partie 2)
}

\author{
Farhad Khosrokhavar
}

Le martyropathe : l'individu-dans-la-mort

2 L'avènement concret de l'individu dans les sociétés soumises à une modernisation rapide s'effectue d'une part par la déstructuration de l'ordre communautaire traditionnel, d'autre part, par l'affirmation de soi d'un être en voie d'atomisation, qui ressent un sourd malaise où entrent en conflit sa volonté de libération et son sentiment d'appartenance à un ensemble qu'il trahit en s'autonomisant. La révolution exacerbe le conflit entre ces deux tendances en introduisant un troisième principe, à savoir l'utopie révolutionnaire et au moment de la rupture de celle-ci, la figure surcharismatique de Khomeyni. Plus le mouvement révolutionnaire se désagrège dans la réalité, et plus le jeune martyropathe a tendance à surcharismatiser Khomeyni pour parer à la déficience du réel. Pour cela, il en fait non seulement la figure de proue au niveau de son individualité et de son sentiment intime d'exister, mais aussi, en relation à la famille et à l'ordre communautaire auxquels il substitue purement et simplement le Guide charismatique. Dans le même temps, ce Guide voit son charisme s'accroître du fait de la nature du message qu'il répand, à savoir, un chiisme mortifère qui sacralise la mort et dénie à la vie toute légitimité ici-bas, dans cette période révolutionnaire où tout se conjugue, pour cette fraction de jeunes en désarroi, sur le mode apocalyptique. Les jeunes martyropathes s'enferment dans Bassidje et se détachent de la société qui les entoure. La rupture des liens sociaux devient à son tour source d'angoisse. Le jeune qui scandait " Liberté, Indépendance, République islamique " au début de la révolution, ne sait plus que faire de sa propre liberté ni de son indépendance d'esprit. Désormais, il est incapable de se prendre en charge, il est dans une situation de plus en plus hétéronome, il vit sa vie dans l'angoisse et la mauvaise conscience, il ressent désormais sa liberté comme une transgression des normes " révolutionnaires " et s'explique les déboires de la révolution à partir de ses propres manquements à ses idéaux. Ceci le culpabilise à mort. C'est pourquoi, à peine délié des attaches communautaires, ce nouvel individu est déjà en crise, dans ce mouvement révolutionnaire où il a investi son identité, et qui l'emporte en déraillant. Le puritanisme exacerbé des bassidji est une expression " moralisée " de ce sentiment de culpabilité qu'ils ressentent d'avoir manqué à l'idéal révolutionnaire, de ne pas s'être investis suffisamment au service de 
l'islam révolutionnaire et d'avoir ainsi entraîné la dérive de la révolution. De même, si au début de la révolution, les jeunes cherchaient à moderniser l'islam en islamisant la modernité, désormais la modernité devient synonyme de péché, de stupre et de manquement à la révolution. D'où la surenchère dans l'archaïsation, par l'adhésion farouche et rancunière à une reformulation de la tradition, elle-même réifiée, déformée et mythifiée. Dès lors, apparaît l'archéo-islam, invention contre-moderne qui se donne pour la tradition mais qui n'est, en définitive, qu'une modernité inversée, où la vie devient la mort, la consommation devient la consomption de soi (on se consomme littéralement dans le martyre qui prend, dans certains cas exacerbés, l'allure d'une homophagie, manducation de sa propre chair). L'archéo-islam est une modernité invertie où la liberté se transforme en une logique de l'interdit absolu, où l'ouverture culturelle se mue en une tentative de se fermer aux autres et ceci, de manière exhibitionniste, c'est-à-dire, paradoxalement, en donnant à voir cette fermeture et en y découvrant un sentiment de participation inversée à la modernité. Les interdits édictés par le Hezbollah ne sont plus les tabous communautaires traditionnels, mais des manifestations politisées des formes culturelles du chiisme, elles-mêmes rigidifiées et maximalisées selon une ligne inflexible et hargneuse d'interprétation.

3 La catégorie du politique prend elle-même un sens trouble: dans la perspective du martyropathe, ce n'est plus un lien entre des êtres autonomes dans l'espace public (démocratie), ni une manière de gérer la vie collective par le recours à des sources sacrées dont l'interprétation serait confiée à des docteurs de la loi (théocratie islamique institutionnalisée) mais une manière de compenser le malaise ressenti par une jeunesse qui ne sait plus comment régir son individualité et qui, par le recours à une catégorie répressive du politique, entend se dessaisir de son être individué et embrasser la mort. La martyropathie devient l'expression ultime du " politique " et en même temps, le moyen légitime par lequel se constitue " l'individu-dans-la-mort ". On voit ainsi se rejoindre l'hyperpuritanisme (expression moralisée de l'interdiction de vivre), l'archéo-islam (le sacré s'investissant dans une religiosité mortifère) et la surcharismatisation du Guide de la révolution (il est la voix " islamique " qui proclame l'inanité du monde et l'accès au sacré par la mort).

4 La mort devient l'issue d'un mouvement où les aspirations s'étaient démultipliées exponentiellement au tout début. Le mouvement révolutionnaire est le catalyseur des désirs et l'opérateur magique de leur réalisation hic et nunc. Le sujet se trouve impliqué dans un engrenage sans issue puisque le désir de se doter d'une nouvelle individualité demeure un projet irréalisable. La mort menace d'emporter le nouvel individu, riche d'un imaginaire modernisé, pauvre à cause de l'impossible réalisation de ses rêves dans une situation de régression économique et d'incertitude sur l'avenir. Dès lors, la sacralisation de la mort résout le problème, puisque, désormais le désir s'investit dans une mort que l'ego parvient à réaliser et il s'autoréalise dans cet acte qu'il accomplit et où il se concilie désormais avec son être tout en préservant son rapport au sacré, à l'islam. Cette mort, à la différence de la mort anomique, n'évacue pas entièrement le sens, mais l'investit là où la réalisation du désir lui-même entraîne la disparition de l'individu.

5 L'aspiration à la modernité du jeune sujet révolutionnaire peut revêtir principalement deux formes: soit la consommation, comme mode de participation festive à la modernité, soit le développement productif, comme mode de participation prométhéenne à la modernité. Dans cet Iran en guerre où la production bat de l'aile, ces 
deux modes de participation se révèlent impossibles. En un sens, ils sont trop prosaïques pour une partie de la jeunesse, avide d'absolu. La révolution se transforme en une quête d'absolu dans une néo-mystique mortifère où tout ce qui tient à la vie se révèle insignifiant, voire insensé. La quête de l'absolu ne saurait se satisfaire que dans l'étreinte de la mort. Le sentiment prométhéen, instillé par la modernisation, se satisfait désormais dans la création mortifère de soi. La conscience révolutionnaire devient le siège de la production de la mort, de la production de soi dans la mort. La consommation, battue en brèche par l'économie de guerre et le chaos social, se fixe à son tour sur le corps : on se consume, faute de consommer, et on transfère le désir de consommation dans un sacré mortifère. Le néo-ascétisme nécrophile en est la conséquence directe ${ }^{1}$.

6 Quelques traits singularisent le jeune martyropathe qui adhère à Bassidje. Pour commencer, il a un univers mental obsidional où sa préoccupation est l'omniprésence des ennemis de l'extérieur et de l'intérieur, ce qui oblitère toute autre forme de considération à ses yeux, y compris les soucis familiaux. Le jeune bassidji a une vision de soi marquée par une grande culpabilité : si la révolution est dans une situation difficile, si les difficultés s'amoncellent de toutes parts, cela est dû au manquement de la société qui n'est pas suffisamment musulmane, et en particulier, à ceux du jeune bassidji lui-même, qui ne parvient pas à se " purifier " jusqu'au bout et à faire de la révolution son souci exclusif dans la vie ${ }^{2}$. Coupable d'impureté, le jeune bassidji vit dans une société encore plus " impure ", encore plus corrompue que lui, ce qui justifie à ses yeux toute forme de répression aux fins de la " purifier " en supprimant les partisans d'une société non-islamique.

7 La martyropathie est un mouvement dont la dynamique suppose, du moins dans les premières années, la jonction du bas (la jeunesse révolutionnaire) et du haut (l'Etat post-révolutionnaire). Elle a ses racines dans la société post-révolutionnaire et dans son instabilité foncière. La manipulation étatique intervient dans une société en effervescence qui a du mal à renouer avec le réel, et où le spectre de la guerre intervient, qui pousse les jeunes à rallier Bassidje, les uns mus par la volonté expresse de mourir au monde, les autres pour défendre la patrie révolutionnaire en danger, les uns (les ludiques) pour s'affirmer sur le champ de bataille, les autres pour s'y éclater, enfin d'autres pour accélérer leur ascension sociale. Cela signifie que l'Etat postrévolutionnaire n'est pas le seul acteur de Bassidje et qu'il est capable d'enrôler les jeunes parce qu'il se trouve à la croisée d'une révolution dont les leitmotiv mobilisent encore les jeunes, les uns pour y exprimer leur désespoir, les autres pour s'acharner à la perte de l'ennemi contre-révolutionnaire. Autrement dit, l'Etat n'est pas l'initiateur exclusif de ce qui deviendra, au fil de la guerre, le " chiisme mortifère "3.

Bassidje porte en lui une détermination essentielle de la nouvelle individualité en gestation chez les jeunes : à savoir sa nature égalitaire dans son symbolisme, dans son mode de recrutement, dans le mode de désignation de ses chefs, et tout aussi bien, dans sa capacité d'assurer aux uns et aux autres l'indifférenciation sociale. Tout ce qui sépare dans la société post-révolutionnaire y est symboliquement nié et voué à l'indistinction. Les jeunes y occultent le cloisonnement de la société en riches et pauvres, " oppresseurs " (mostakbar) et " déshérités " (mostaz'af), supérieurs et inférieurs, pratiquants et non-pratiquants : la pratique de la guerre sainte se substitue implicitement comme forme de prière privilégiée et on y est traité en musulman, même 
si on ne pratique pas assidûment ses prières quotidiennes sur lesquelles on n'est pas très exigeant à Bassidje.

9 Toute hiérarchie extérieure est abolie, on y pratique une " hiérarchie du cœur ", les différences s'y noyant dans un sentiment d'appartenance unitaire et égalitaire: les chefs sont aussi des bassidji, ils ne pratiquent pas la distance que les gradés de l'armée traditionnelle maintiennent vis-à-vis des soldats, ils n'hésitent pas à être sur les premières lignes de combat. L'effacement de la hiérarchie et de la discipline contribue au sentiment unitaire, même si, par ailleurs, la hiérarchie y existe bel et bien, les considérations de " fiabilité " des dirigeants (leur identification à la République islamique) y entrant pour beaucoup après les premiers échelons.

Le rapport à la hiérarchie n'est pas le même selon que l'on est martyropathe, ludique ou opportuniste. Le jeune martyropathe renonce à son identité au profit de l'organisation qui lui confère la plénitude dans la mort, il se livre littéralement à la hiérarchie parce qu'il entend se défaire de son désir de vivre de manière autonome. Bassidje satisfait ainsi à l'aspiration des martyropathes, à l'hétéronomie. Par contre, chez les ludiques et les opportunistes, c'est l'effacement de la hiérarchie, l'égalitarisme et le sentiment de fraternité qui sont attrayants. A une société inégalitaire, le jeune oppose l'égalité fraternelle de cette organisation où prévaut une idéologie égalitaire face à la mort. Chez l'ensemble des jeunes, l'éventualité de mourir en martyr donne lieu à un Nous égalitaire et pur (" sans déshonneur "), en rupture par rapport à un Eux (la société post-révolutionnaire) inégalitaire et pervers (impur).

11 Bassidje parvient à jouer sur deux registres, incompatibles dans la société. Aux martyropathes, elle donne le sentiment de la hiérarchie, de l'Ordre total et sacré. Aux jeunes ludiques ou à ceux qui recherchent la rupture avec une vie quotidienne inégalitaire et injuste, elle satisfait au besoin de rupture avec la pauvreté et la situation dégradante du déshérité, et donc au besoin de l'absence de hiérarchie. Elle y parvient par le recours constant à la figure charismatique de Khomeyni qui cautionne la légitimité de Bassidje par sa sanctification. Pour les jeunes martyropathes, Bassidje n'est pas une organisation comme une autre, mais le lieu de la prise en charge de soi par Khomeyni, le site où la rencontre avec l'Imam du Temps s'effectue par le truchement du Guide de la révolution ${ }^{4}$. Dans la société réelle, ces deux logiques sont éclatées depuis la décomposition du mouvement révolutionnaire qui, pour un moment, avait réussi à les relier dans l'unanimité du début. Bassidje, par la manipulation symbolique des jeunes, préserve artificiellement cette ambiance unitaire où ces deux ordres de faits s'associent dans l'irréalité d'une fraternisation à huis clos. Mais, s'il y a manipulation par l'Etat des jeunes, c'est parce que la conjoncture post-révolutionnaire la permet : cet Etat n'est ni celui qu'il deviendra quelques années plus tard (l'emprise d'une domination sans légitimité), ni celui du début de la révolution (expression de l'unanimité au sein de la société), mais un entre-deux qui dissimule et occulte la rupture de l'unanimité révolutionnaire. Les jeunes continuent à rêver à cette unanimité des cœurs au sein d'une société où celle-ci a disparu, et puisent dans la guerre et au sein de Bassidje le moyen de refuser une réalité à laquelle ils ne peuvent plus souscrire : plutôt mourir à la vie que d'avoir à renoncer à une logique de rêves qu'enflammait la révolution et contre laquelle, la réalité qui se dresse est désormais perçue comme un ennemi à abattre. Le martyre révolutionnaire contribue à retarder le désespoir face à une réalité cruelle, mais il le nourrit en un autre sens, en lui donnant un contenu sacré. 
12 La religiosité des martyropathes, on la qualifiera de chiisme mortifère. Elle est différente autant du chiisme communautaire traditionnel que du chiisme de l'aube de la révolution, gonflé par l'espérance utopique de la nouvelle société islamique à construire. Le chiisme mortifère est une représentation où la mort exerce une fascination irrésistible non pas pour édifier une société nouvelle, pour réaliser une utopie, mais en désespoir de vivre dans un monde déserté par l'espérance, monde où le sujet se sent de plus en plus comme un étranger et où il exprime son désarroi par un passage à l'acte qu'il sacralise sous le nom du martyre. Cette mort sacrée est pourtant marquée par une différence essentielle par rapport au martyre chiite traditionnel. D'une part, il s'agit de mettre en œuvre sa mort, voulue d'avance (alors que dans le martyre traditionnel, le combattant de la foi cherche à réaliser l'idéal religieux et qu'il succombe à la mort, involontairement, en se sacrifiant pour cet idéal). En second lieu, ce néo-martyre est la réalisation de soi dans la mort en tant qu'individu en gestation. Alors que le martyre traditionnel est vécu comme un événement archétypique et à la limite non reproductible (seul l'imam Hoseyn ${ }^{5}$ et une poignée d'élus y ont eu droit, pas le commun des mortels), à présent, tout musulman y a accès, le martyre " se démocratisant " dans le même mouvement où le religieux se sécularise. La martyropathie, forme désespérée d'expression de soi de l'homo islamicus novus, est la conséquence de l'introduction du sujet dans une modernité traumatisante où les structures intermédiaires qui empêchaient la mise à mort de soi ont subi un intense processus de désarticulation. Ceci nous renvoie à la troisième caractéristique de ce type de martyre, à savoir, sa dimension suicidaire. C'est par désespoir de vivre, dans une situation vécue comme intolérable (le moment de l'échec de l'utopie dans le cas iranien, le moment de la reconnaissance par le jeune Palestinien de l'absence de solution concrète à ses problèmes de vie sous occupation israélienne...), que le jeune a recours à la martyropathie, pour mettre fin à ses jours, sous prétexte du sacré. Mais ce faisant, il l'accomplit en s'occultant la dimension suicidaire de son acte. Il sacralise sa mort en tentant de construire une véritable communauté dans la mort. Ne pouvant vivre la positivité d'une modernité dont il sait qu'elle existe concrètement ailleurs, il se la donne en déniant à la vie la légitimité, en noircissant le monde moderne où il vit dans l'exclusion et il érige une modernité à l'envers dans une nécro-communauté qui n'est pas du tout celle de la communauté islamique traditionnelle. Dans la martyropathie, il y a d'une part une véritable fascination pour la mort (elle constitue paradoxalement la seule espérance qui reste au sujet) et d'autre part, l'aspiration individualisée à mourir comme unique mode d'expression d'un Ego qui entend désormais se réaliser dans la mort. Pour cela, le sujet a besoin d'une véritable " communauté dans la mort " qui lui donne le sentiment de ne pas être seul dans sa tâche sacrée à réaliser. Dans le cas iranien, Bassidje remplit cette tâche, dans les autres cas, des groupes plus ou moins restreint accomplissent cet office. Autant l'individu déstructuré, désespéré, imprégné d'une eschatologie mortifère (religiosité mortifère) est la condition sine qua non pour la réalisation de ce mode d'action suicidaire, autant l'existence d'un groupe ou d'un groupuscule qui prend en charge la sociabilité désespérée du jeune est d'importance, pour que le sujet ne se cantonne pas à ruminer son désespoir et cherche à se réaliser dans la mort en déclarant la guerre contre le monde ambiant où il se sent malheureux. La religiosité mortifère a la capacité de transformer le monde, en ennemi à abattre, et c'est ce qui la différencie du désespoir anomique. Dans cette forme de désespoir, le sujet n'entend pas mourir silencieusement, mais entraîner le monde dans son sillage : autant il lui est " vital " de mourir, autant il 
lui faut imprimer sa marque sacrée sur un monde dont la seule raison d'être est son annihilation violente pour réaliser le vœu du sujet: mettre un terme à la vie qui devient le dernier site où l'impureté se niche. Désormais, la vie est impure, et la mort, forme ultime et sacrée de réalisation de soi, apporte l'exclusive possibilité de réalisation de soi dans la " dignité ". La martyropathie post-révolutionnaire iranienne relève de cette religiosité mortifère où entrent en jeu un désespoir profond et la tentative de le voiler et de le surmonter oniriquement par le recours à une religiosité rendue activement nécrophile.

Un autre trait distinctif des martyropathes est leur profonde instabilité. Ils oscillent entre deux principes contradictoires: d'une part, celui de l'Ego révolutionnaire qui s'affirme dans la défense de l'utopie révolutionnaire en combattant ceux qui s'y opposent (la conscience révolutionnaire classique), d'autre part, celui de l'abdication de la vie et la fuite dans le suicide par désespoir de ne pouvoir se réaliser dans le monde. D'où, l'urgence de rompre avec la vie, dernier vestige de " l'impureté ". Cette dimension est profondément mortifère : le jeune martyropathe est attiré par la mort, par la sienne mort qu'il entend réaliser en entraînant les autres dans son sillage. Dans cette dimension léthifère se manifeste la " nouveauté " de la mentalité révolutionnaire iranienne : on entend autant faire mourir l'ennemi que soi-même, l'un et l'autre étant équivalents dans la perspective où se placent les bassidji nécrophiles pour qui la vie n'a plus de sens dans ce monde.

14 L'état mental où coexistent ces deux composantes (l'affirmation de l'Ego dans l'utopie révolutionnaire et sa démission dans le suicide) singularise le martyropathe qui oscille constamment entre les deux pôles et dont l'instabilité foncière engendre une vision agonistique du monde et une représentation obsidionale de soi.

Cette conception de soi sur fond d'un désespoir sacralisé est en même temps une modalité de la sécularisation du religieux: désormais, tout jeune peut prétendre rejoindre la horde des compagnons sacrés de l'imam Hoseyn, devenir un saint en miniature, et rompre ainsi la vision aristocratique de la religiosité traditionnelle où seulement une poignée de croyants (dans le cas de Hoseyn, seulement 72 compagnons) avait droit au privilège du martyre; en second lieu, le religieux n'est plus l'expression doloriste d'une identité " hors du monde " qui se réaliserait par des rituels normés (les processions flagellantes du Tasou'a et d'Achoura où se célèbre chaque année l'anniversaire du martyre de Hoseyn), mais dans la proximité même de la vie quotidienne, sur le front de guerre, ou dans la lutte contre les adversaires de la République islamique, dans la rue, en plein milieu urbain, au jour le jour. Le religieux se sécularise dans la mesure même où le sujet s'individualise ou plus simplement, s'individue. Mais on passe alors directement de " l'individu hors du monde " du chiisme traditionnel à " l'individu-dans-la-mort " de la martyropathie ${ }^{6}$.

Le sombre désespoir du martyr fait qu'il perçoit la vie dans le monde comme une forme d'impureté, et qu'il est convaincu de la nécessité de la mort pour se purifier. Dans cette attitude se donne à voir l'hétéronomie de l'Ego, instable et oscillant entre l'affirmation de soi dans la lutte acharnée contre les " ennemis " et sa fuite dans le suicide. Pareille ligne de conduite induit la rupture avec la société où la vie dans ses actes les plus insignifiants est une insulte aux convictions mortifères du martyropathe. Ainsi, en permission pour quelques jours auprès de sa famille, il abrège souvent son séjour et quitte femme, enfants ou parents pour se rendre sur le front, le spectacle de la vie dans 
la Cité, parmi des gens tenant " lâchement " à la vie (expressions qui abondent dans les testaments), lui répugnant profondément.

Un problème crucial chez le martyropathe est son rapport au sacrifice. Il croit se sacrifier pour l'islam, en réalité, il ne fait que céder à un désespoir brut. Le sacrifice du martyr prend son sens dans la mesure où il est partagé par les autres. Dans la société iranienne en pleine révolution, les jeunes qui accédaient au martyre étaient perçus par l'immense majorité des gens comme se " sacrifiant " pour l'islam, ce sentiment étant partagés par eux-mêmes. Par contre, les Bassidji, eux, pensent " se sacrifier ", là où les parents et les autres pensent qu'il s'agit d'une attitude inconséquente pour soi, et répressive pour les autres (le martyre du jeune Bassidji est monnayé par le pouvoir pour se légitimer sur le plan politique et faire perdurer une guerre qui devient de plus en plus sanglante et de plus en plus onéreuse, sans aucun dénouement sur le terrain, à partir de la troisième année). Le sentiment du sacrifice du jeune n'est donc pas partagé par les autres, et c'est cet " aveuglement " qui lui permet d'agir comme s'il représentait les autres, alors que la grande majorité a déjà renoncé à l'utopie révolutionnaire et n'est plus à l'unisson avec le pouvoir hezbollah qu'il défend et que les autres rejettent sans vouloir ou pouvoir se mobiliser contre lui. Le martyropathe est ainsi ce révolutionnaire attard,é qui agit comme si l'unanimité des cœurs était préservée, lors même que depuis la deuxième année de la révolution, celle-ci a volé en éclats, et que dans son attitude même, le jeune révolutionnaire reproduit cette scissiparité ; il agit soi-disant pour réaliser l'utopie révolutionnaire, en réalité, il n'est là que pour sceller l'échec de la révolution : celle-ci n'est pas susceptible de se réaliser dans ce monde?

L'individu-dans-la-mort qu'incarne le martyropathe est le fait d'une minorité de jeunes dont la religiosité nécrophile revêt pourtant une grande importance tout au long de la guerre: le pouvoir hezbollah en fait le fer de lance pour légitimer son ascendant répressif sur la société et pour s'en prévaloir aux fins de promouvoir un néopuritanisme où une morale rigoriste se lie avec une vision politique fermée. La grande majorité, tout en ne souscrivant pas à cette vision nécrophile de l'existence promue par les martyropathes de Bassidje, ne réagit pas non plus frontalement à son encontre et ne lutte pas ouvertement contre un pouvoir qui s'impose par la terreur. La voie qu'elle choisit est celle de la mise en place d'une double identité : d'une part, celle que l'on offre en pâture au régime islamiste, à savoir l'apparente soumission à son idéologie dans l'espace public; d'autre part, la constitution de leur individualité dans un lieu que ne contrôle pas entièrement le pouvoir, à savoir le marché noir. Le pouvoir a beau revendiquer la lutte à mort contre l'impérialisme américain (le Grand Satan), dans le marché noir qui s'étend de plus en plus sur la société, le dollar règne sans partage. L'idéologie officielle est basée sur l'abnégation absolue du sujet martyropathe, le marché noir est le lieu d'un égoïsme effréné où se manifeste, sous une forme sauvage, l'individu dans le lucri rabies. Par ailleurs, le marché noir est un démenti cinglant au régime et à ses promesses du début : l'inflation galopante, l'apparition des stockeurs et des accapareurs qui usent d'une économie de guerre et de pénurie pour s'enrichir de manière fulgurante, avec la complicité des agents du pouvoir,et de la jeunesse opportuniste qui collabore avec le Hezbollah. On vit de plus en plus dans une société éclatée, voire même schizoïde où, d'un côté, les martyropathes prônent la mort et aspirent à y accéder (individu-dans-la-mort), et d'autre part, de larges pans de la société exercent des activités plus ou moins louches et illégales au sein d'un marché noir qui devient le lieu de l'affirmation de l'individu dans la vie, cette fois sous une forme sauvage et effrénée, forme dominante de la gestion de la quotidienneté dans la 
société post-révolutionnaire. Cette dualité, le martyropathe la vit de manière douloureuse : il ne supporte pas que les idéaux de la révolution soient bafoués aussi aisément, que le réel puisse annihiler à peine deux années après la révolution, l'utopie de cet islam révolutionnaire qui aurait dû instaurer la justice et la fraternité dans la société. Cette distance de plus en plus croissante entre ses rêves et la réalité occasionnent un désespoir qui débouche aisément sur la mort.

Un puritanisme exacerbé singularise la révolution iranienne après la prise de pouvoir par le Hezbollah, à peine un an après le renversement du pouvoir du chah. Certes, toutes les révolutions sont plus ou moins puritaines, mais dans le cas iranien, le puritanisme est poussé à un degré extrême. Celui-ci a partie liée à l'hégémonie du Hezbollah, qui puritanise les relations sociales comme une forme supplémentaire d'intimidation et de répression à l'encontre d'une société qui vient de goûter au court intermède révolutionnaire où la liberté d'expression n'était pas un vain mot. Le pouvoir hezbollah impose des normes puritaines à la société et entend, au nom de l'islam, joindre la légitimation morale et la répression politique des opposants, créant ainsi un espace amphibologique, où, tout opposant est nécessairement immoral et tout être immoral, un opposant " politique ". En réalité, le Hezbollah condamne l'hédonisme ou la recherche du plaisir comme signes de volonté d'autonomie par rapport à sa conception totale du pouvoir.

Le puritanisme post-révolutionnaire a une autre composante, celle-ci liée au consumérisme introduite par le régime impérial à partir du début des années soixantedix en Iran. La jeunesse hezbollah consomme du puritain, pour une raison patente : si sous le régime du chah la consommation était vécue dans l'abondance pour les classes moyennes et supérieures ou sous forme de rêve d'abondance pour les classes inférieures, le néo-puritanisme est lié à une période de pénurie (la guerre, le déclin de l'économie) où on ne peut plus consommer que du symbolique, faute de réel. Mais, la même démesure, la même volonté d'appropriation des choses et la même soif inextinguible habite le sujet. Cependant, le néo-puritanisme hezbollah n'est pas uniquement l'adhésion au consommatoire. C'est le refus de plus en plus hargneux de la modernité consumériste par une jeunesse révolutionnaire désespérée qui s'enferme dans le mythe d'une communauté archaïque. Le puritanisme dicte un type de comportement qui veut mettre fin au règne d'une modernité impure parce que consumériste, consumériste parce qu'impure, pour embrasser le pur dans l'interdiction, et le cas échéant, dans la mort. La modernité est saisie comme une impureté, comme la transgression d'un islam préalablement puritanisé pour pouvoir servir de bouclier contre celle-ci.

21 Mais, le puritanisme post-révolutionnaire a aussi partie liée avec la crise de la nouvelle société. L'attitude néo-puritaine des jeunes qui sévit après la révolution n'est pas la réplique $\mathrm{du}$ comportement traditionnel en matière $\mathrm{d}$ 'honneur communautaire (nâmous) et d'honnêteté féminine. Jadis, ces conduites étaient intériorisées et la loi coutumière ou la jurisprudence islamique apportaient leur sanction à des normes que respectait l'écrasante majorité des musulmans. A présent, la proximité sexuelle liée aux nouveaux modes d'urbanisation et les bouleversements opérés par le changement social remettent en cause le respect inconditionnel des normes islamiques traditionnelles. Les jeunes sont dans un mouvement contradictoire et conflictuel, d'une part, fascinés par les relations (imaginaires ou réelles) avec l'autre sexe, et d'autre part, minés par le remords de transgresser les normes islamiques. Avant, la communication 
existait surtout entre les hommes, les rapports homme/femme étant régis par un code qui excluait, dans sa tendance centrale, l'intimisme et l'amour ${ }^{8}$. Aujourd'hui, dans les relations entre les jeunes, transparaît un sensualisme latent qui a peur de s'exprimer sous son vrai jour et qui se travestit sous de multiples formes: par exemple, dans les groupes d'extrême-gauche, par des séances d'endoctrinement idéologique, où hommes et femmes nouent des relations réciproques, au contenu crypto-sexuel. C'est pourquoi le puritanisme du Hezbollah trouve un terrain plutôt favorable dans de nombreuses couches populaires, récemment modernisées, leur enracinement dans le monde rural (les paysans dépaysannés) et leur déracinement dans les années soixante suite à la Réforme agraire du chah, créant chez eux le sentiment de vivre dans une ville où règnent le stupre et la débauche. Le rigorisme exacerbé de Hezbollah trouve ainsi un écho plutôt favorable dans ces nouvelles couches populaires, en voie de modernisation dans l'espace urbain des grandes villes. Au malaise existentiel des martyropathes s'adjoint le sentiment de dépossession de soi en matière d'honneur chez les paysans dépaysannés9. Ceci donne aux jeunes martyropathes un sentiment accru de légitimité et renforce le pouvoir hezbollah.

Crise d'autorité dans la famille

La famille traditionnelle est mise à mal par la modernisation sauvage de l'ère impériale qui brise une part de ses réseaux de solidarité par la substitution de l'Etat à l'ancienne communauté dans de nombreux domaines. Elle l'est aussi par l'Etat postrévolutionnaire qui introduit momentanément un principe d'autorité supérieure, celle de Khomeyni comme " père au superlatif " qui empiète sur la fonction du pater familias dans tous les domaines où se trouve mise en jeu la défense de l'islam politique qu'il prône. Elle l'est enfin par les jeunes martyropathes qui marquent leur prédilection pour la religiosité mortifère que prône le Guide de la révolution et abandonnent souvent la famille pour aller rejoindre Bassidje. Si l'Etat islamiste remet en cause, de l'extérieur, l'autorité du père, le martyropathe, lui, sape de l'intérieur ce pilier de l'ordre anthropologique dans la société. La perte du sens de la famille est l'un des signes les plus patents de la religiosité martyropathe exacerbée. Père, mère, amis, frères, le martyropathe finit par les perdre de vue en se vouant à sa passion mortifère qui, en s'exacerbant, devient son unique et exclusive préoccupation.

Petit à petit, sous l'influence de l'idéologie nécrophile de Bassidje, des prônes des mollahs " made in Bassidje " et de l'atmosphère générale qui règne au sein de cette organisation, le martyropathe se trouve dans l'incapacité de concevoir l'existence hors de sa passion mortifère. Bassidje fait ici office de quasi-secte, d'ensemble clos, porté par une effervescence qui s'entretient, se reproduit, voire même s'exacerbe dans le tête à tête de ceux de ses membres qui visent la mort comme ultime phase de leur purification spirituelle, avec l'image de Khomeyni Guide suprême en tête. Dès lors, s'estompe l'image du père, de la mère, des parents et des connaissances, tout perdant son sens dans ce rapport exclusif à une mort dont la réalisation devient l'exclusif moyen d'ériger un sens. La passion du martyropathe peut l'entraîner à rechercher la suppression physique de la famille, de la sienne comme de celle des autres. Dans un premier temps, son souci majeur est qu'après sa mort, sa famille se comporte non pas comme une famille en deuil, mais " en liesse ". Mais sa passion mortifère s'accusant, il en vient quelquefois à lui demander de lui emboîter physiquement le pas, à participer à sa mort comme dernier témoignage de l'adhésion à la Vérité. Si la famille, dans sa lignée mâle, y acquiesce, on est face à une famille martyropathe (cf. Infra). Dans ce cas, 
ce que demande le martyropathe à sa famille, ce n'est pas tant de se comporter en digne famille de martyr après sa disparition, mais de mourir à l'essence de la famille en renonçant au souci de préservation de ses membres. " Individu-dans-la-mort ", il peut aller jusqu'à rejeter cette famille qui voudrait préserver sa vie, en le détournant de gré ou de force de sa passion mortifère. Quoiqu'il en soit, face à ses exigences exorbitantes, l'appui qu'il reçoit de l'Etat khomeyniste réduit, dans la plupart des cas, la famille à un statut secondaire aux marges de manœuvre fort limitées.

Dans la représentation du martyropathe, le locus du péché n'est pas la famille (on ne se culpabilise nullement d'abandonner de vieux parents, quelquefois femme et enfants, en partant sur le front vers une mort quasi-certaine), mais, l'ordre islamiste, à l'égard duquel, le martyropathe craint de manquer de dévouement, même en lui faisant don de sa vie. Cela signifie que dans sa psyché déstructurée, l'ordre islamiste se substitue à la famille.

Comme si sa mort n'amputait pas suffisamment sa famille de l'un de ses piliers, il prêche la mort collective au service de son sacré mortifère. Le martyropathe cherche moins à mettre son ardeur de vivre au service de la guerre qu'il ne se saisit de la guerre pour offrir un exutoire à sa soif de mourir.

Dans la psyché déstructurée du martyropathe, il y a annexion du rôle du père par Khomeyni, chez qui le sujet découvre le catalyseur de son ardeur de mourir.

Dans le même temps, Khomeyni isole les jeunes qui collaborent avec le nouvel ordre répressif du reste de la société en les enfermant dans Bassidje, ce qui les fait honnir de la société. Le foisonnement des organisations répressives après la révolution permet un encadrement progressif de la société, même dans les zones rurales, que ce soit de manière directe (les Comités, l'Armée des Pasdaran, la Savama qui se substitue à la Savak en gardant une bonne partie de son personnel), soit par le recours aux fondations pieuses ou les structures para-étatiques (Fondation du 15 Khordad, Fondation des Martyrs, Croisade pour la Construction - qui sera ultérieurement intégrée dans un ministère). Ces organismes permettent à une partie des jeunes d'y adhérer et d'y investir cette part de leur identité qui n'arrive plus à s'investir dans la société, vu sa déstructuration de plus en plus poussée, surtout en zone urbaine. Les organismes de répression (le Comité, l'Armée des Pasdaran, la Croisade pour la construction, la Croisade universitaire...) quadrillent la société, mais, ils pourvoient aussi la jeunesse hezbollah d'un sentiment d'identité " islamique ", en institutionnalisant son attachement à Khomeyni. Dans une société en crise politicoéconomique, secouée par la guerre et en butte à une révolution qui déstabilise les esprits, la volonté d'autonomie des jeunes vis-à-vis de la famille entraîne l'adhésion plus ou moins forcée des jeunes à l'Etat, tant pour des raisons matérielles qu'affectives et clientélistes.

Dans cet Iran en pleine tourmente révolutionnaire, le sujet martyropathe n'est pas la seule expression de la crise sociale. Un autre phénomène voit le jour, statistiquement beaucoup plus limité mais sociologiquement doté d'importance, à savoir, la famille martyropathe. Celle-ci est une configuration originale dans les familles urbaines, tout au long de la guerre. Elle se différencie de la très grande majorité des familles par trois traits. Tout d'abord, au lieu que la famille s'oppose au départ de ses jeunes fils au front, comme cela se produit dans la grande majorité des cas, elle les y encourage vivement, non pas pour des raisons financières ou clientélistes, mais par conviction mortifère. En second lieu, non seulement les jeunes fils partent sur le front, mais aussi le père, voire 
l'oncle ou le neveu qui leur emboîtent le pas. Enfin, les femmes elles-mêmes partagent souvent cette conviction mortifère et interviennent pour convaincre fils, frère ou père, de partir sur le front en adhérant à Bassidje.

On peut distinguer plusieurs configurations dans la famille martyropathe : quelquefois, ce sont uniquement le père et certains fils qui optent pour la martyropathie. C'est le degré minimal de famille martyropathe. Quelquefois, ce sont trois générations d'hommes (grand-père, père et fils ou grand-oncle, père et neveu ou grand-oncle, père et fils avec toutes les combinaisons possibles), qui rejoignent Bassidje pour accéder au martyre mortifère. Dans certains cas, les femmes poussent à leur tour les hommes à s'engager dans Bassidje. Dans ce cas maximaliste, tous les tabous sociaux (le caractère insupportable de la mort du fils et du chef de famille) sont surmontés dans un élan mortifère ${ }^{10}$. On reconstitue l'unanimisme révolutionnaire au sein de la famille par le départ collectif de la lignée mâle (père et fils) au front. On se retrouve dans Bassidje, l'unanimité se refaisant, par-delà les clivages dus à la rupture des générations; tout le monde échappant à la rupture de l'unanimité révolutionnaire dans une ambiance de fraternité morbide, dénonçant le monde et ses turpitudes au nom d'une pureté mortifère.

Dans la famille martyropathe, la disjonction mentale des membres de la famille avec la réalité ambiante est beaucoup plus poussée que dans le cas général où le fils, en ralliant Bassidje, rompt avec la famille. Dans le dernier cas, la famille maintient son contact avec le réel en préservant la vie en son sein, tout en s'endeuillant de la mort du fils. Dans la famille martyropathe, le " décollage " ne concerne plus uniquement le fils, mais les hommes de la famille (plusieurs jeunes fils, mais aussi le père, l'oncle etc.). Dans ce martyre collectif, les parents comblent le fossé des générations pour devenir complices des jeunes dans une mort partagée qu'ils goûtent comme la reconstitution de l'unité familiale, à l'abri de la déstructuration traumatisante de la société, de l'unité révolutionnaire et des dissensions post-révolutionnaires ${ }^{11}$.

De même qu'il y a des familles particulièrement enclines à la martyropathie, de même certaines régions se caractérisent par un nombre élevé de vocations martyropathes. Parmi elles, on peut citer Nadjaf-âbâd dans la province d'Isfahan où de nombreuses familles ont " fait don " de plusieurs martyrs et quelquefois, de tous leurs fils à la révolution. Plusieurs explications peuvent être avancées pour rendre compte de ce phénomène qui affecte donc une ville située à une vingtaine de kilomètres de la ville d'Isfahan qui a été l'un des pôles du développement et de l'industrialisation sous le régime du chah. Historiquement, la province d'Isfahan a été le centre officiel de la propagation du chiisme en Iran, après son instauration comme capitale des Séfévides au XVIIe siècle. D'autre part, tout comme nombre de villes iraniennes qui se définissent en opposition symbolique vis-à-vis d'autres agglomérations urbaines voisines, l'antagonisme entre Nadjaf-âbâd et la ville d'Isfahan est notoire. Les habitants d'Isfahan sont caractérisés par ceux de Nadjaf-âbâd comme calculateurs, hypocrites et " radins ", alors que ceux de Nadjaf-âbâd se considèrent comme sincères et généreux. La ville de Nadjaf-âbâd tenterait ainsi de prouver sa supériorité morale sur la capitale régionale, Isfahan, dans un Potlach symbolique, par martyrs interposés. Ce qui, dans la vie quotidienne, était du ressort du symbolique devient, pendant la période de vogue du martyre, un fait réel. Enfin, l'ayatollah Montazéri, dauphin de l'imam Khomeyni avant son limogeage, est originaire d'un village de Nadjaf-âbâd, la participation intense de 
cette ville et de son espace rural à Bassidje donnant un gage de fidélité clientéliste au régime par le truchement de Montazéri.

Dans les grands centres urbains, Bassidje est un organisme essentiel pour canaliser le désespoir des jeunes sur une voie mortifère, en opposition à la ville, à la société. Khomeyni monnaye l'animosité des jeunes à la société pour les attirer vers lui. La jeunesse révolutionnaire se venge de cette société, profondément injuste, cynique et " égoïste ", en s'affiliant à Bassidje. Par contre, dans les petites villes où la cohésion communautaire n'a pas été profondément entamée, la jeunesse n'éprouve pas de hargne contre la société, elle y adhère encore, la solidarité communautaire n'ayant pas entièrement cédé au sourd antagonisme de classes que nourrit l'immersion dans les grandes zones urbaines. Bassidje est nécessaire là où la ville abandonne totalement la jeunesse des couches populaires, dans ces grands centres urbains où celle-ci n'éprouve plus aucun sentiment de solidarité avec le reste de la société, où les " déshérités " se sentent injustement exclus. Dans ces zones, Bassidje est le lieu de germination d'un sentiment d'identité en rupture avec la société. Par contre, dans les petites communautés à forte homogénéité culturelle, la menace contre l'identité communautaire risque de la faire basculer dans la mort, afin de sauver l'identité collective, peu entamée par la modernisation.

Globalement, dans les régions où la modernisation sous le chah a entamé la structuration communautaire, c'est l'individu martyropathe qui émerge, marquant la fin de l'ordre communautaire et l'avènement de " l'individu-dans-la-mort " comme forme sauvage et désordonnée d'un mode de gestion de l'identité où le sujet se prend en charge dans la mort, constituant, dans le même mouvement, l'Etat comme pôle d'identité néo-communautaire. Ce sont des régions comme Téhéran ou Isfahan qui semblent être le théâtre de la martyropathie individuelle, les grands centres urbains étant le théâtre même de ce nouvel être individué qui émerge à l'horizon. Par contre, dans des zones où l'ordre communautaire est plus ou moins vivace et où il a été entamé par la modernisation sans être irréversiblement désarticulé, la famille martyropathe (et non l'individu) prend de l'importance. Les régions qui n'ont pas été intensément modernisées et qui ont été à l'abri des transformations introduites par le régime impérial marquent une grande tiédeur, vis-à-vis de la révolution en général, et de la martyropathie en particulier. Tel est selon toute vraisemblance, le cas du Baloutchistan et du Sistan au sud-est de l'Iran, et du Kerman au sud. Dans ces régions, les jeunes candidats martyropathes sont bien moins zélés qu'ailleurs. Plus la modernisation a atteint les équilibres sociaux et voué l'ordre communautaire traditionnel à la désarticulation, plus la probabilité d'émergence d'une jeunesse martyropathe est grande ${ }^{12}$.

\section{NOTES}

1. Cf.infra, la section sur la néo-mystique mortifère. 
2. Les testaments des bassidji fourmillent de ces considérations. Ils sont publiés tout au long de la guerre avec le soutien actif de la presse et des nombreuses publications de Bassidje et des organismes affiliés, mais le fond de désespoir qu'ils dénotent est authentique et a pu être observé sur le terrain. Cf. L'islamisme et la mort, op. cit., Eric Butel, Le martyre révolutionnaire : stratégies et discours néocommunautaires de la jeunesse populaire iranienne, DEA, INALCO-EHESS, 1995 ; Alain Chaouli, Un groupe minoritaire d'adolescents révolutionnaires iraniens : les Bassidji, mémoire pour le diplôme de l'EHESS, 1996.

3. Cf. L'islamisme et la mort, op.cit., par ailleurs, ceci se voit clairement chez les "correcteurs" de Bassidje. Les "correcteurs" embauchés par Bassidje mettent en forme les testaments des martyrs. Pour ce faire, ils s'inspirent du chiisme mortifère des jeunes. Les testaments, même remaniés par les correcteurs de Bassidje, reflètent l'aspiration à la mort des jeunes dont ils se font les truchements.

4. D'où les nombreux récits de vie des martyrs ou des nouvelles rédigées par eux où on voit, sur le front de guerre, surgir un vieillard illuminé qui peut être indistinctement l'Imam du Temps ou Khomeyni, son représentant. Cf. Pour ces exemples, L'islamisme et la mort, op.cit., et Eric Butel, op.cit.

5. L'imam Hoseyn, le troisième imam chiite, est le parangon du martyr : Prince des Martyrs, il succombe à la mort avec ses 72 compagnons, lors d'une bataille inégale le 10 octobre 680 contre l'armée envoyée par le calife omeyyade Yazid, à qui il a refusé de faire allégeance. Cet épisode, célébré chaque année dans les journées de deuil de tâsou'â et d'âchourâ, forme l'un des piliers de l'identité chiite. Cf. Mahmoud Ayoub, Redemptive Suffering in Islam, The Hague, 1978 ; Jean Calmard : " Le patronage des ta'ziéh : Eléments pour une étude globale " in Ta'zieh, ritual and drama in Iran, edited by Peter J. Chelkowski, 1979, New York University, New York; George Thaiss, "Religious symbolism and social change : The Drama of Husain" in Scholars, Saints and Sufis, edited by Nikki R. Keddie, University of California Press, Los Angeles, 1972.

6. Louis Dumont distingue l'individu hors du monde de l'individu dans le monde à partir de l'expérience de la société traditionnelle indienne. Dans le cas iranien, la sécularisation du sacré induit une forme d'individualité que nous appellerons " l'individu-dans-la-mort ". Cf. Louis Dumont, Essais sur l'individualisme, Seuil, Paris, 1983.

7. Olivier Roy analyse, selon une perspective comparatiste, l'échec des mouvements islamistes. Cf. Olivier Roy, L'échec de l'islam politique, Seuil, Paris, 1992.

8. Cf. Fatima Mernissi, Le harem politique. Le Prophète et les femmes, Paris, 1987.

9. Cf. dans Le discours populaire de la révolution iranienne, tome 2, op. cit., les entretiens, notamment avec Sakiné, fille de paysan dépaysanné, et Hassan K., paysan dépaysanné.

10. De nombreux cas sont relevés dans les mass media comme celui de cette famille, originaire d'Arâk, mais vivant depuis une génération dans les banlieues de Téhéran. Des cinq fils, deux sont morts en martyrs et les trois autres ont été grièvement blessés sur le front. L'un s'est brisé l'épaule, l'autre s'est rompu la colonne vertébrale et demeure paralysé pour le restant de ses jours, le troisième est amputé des deux jambes. Le père, quant à lui, se trouve au front, en volontaire, en 1987. Les journaux abondent en exemples ; par exemple Résâlat, 28.1.1987, p.1-2 qui fait état de quatre fils d'une famille, morts en martyrs (l'un d'eux est porté disparu).

11. La famille martyropathe est à rapprocher des sectes qui tentent quelquefois de commettre un suicide collectif comme Waco aux Etats-Unis (1992) ou la secte du 
Temple du Soleil en Suisse (1994). Ce type de structures est destinée à se démultiplier dans l'avenir, au sein d'une modernité déstructurante.

12. On ne dispose pas de statistique fiable sur le nombre des martyrs par régions, départements et villes. L'analyse ne peut dès lors que se baser sur l'observation individuelle et sur les données qualitatives.

INDEX

Mots-clés : enfants, forces paramilitaires, kamikaze (missions suicide), conflits internationaux Index géographique : Iran

Thèmes : Bassidje, Pasdaran 